\title{
Neuronal ultrastructure is preserved by fructose-1,6-bisphosphate after hypothermic circulatory arrest in pigs
}

Timo Kaakinen, MD, ${ }^{a}$ Anita Naukkarinen, MSc, PhD, ${ }^{b}$ Hannu Tuominen, MD, PhD, ${ }^{c}$ Pekka Romsi, MD, PhD, ${ }^{a}$

Matti Nuutinen, MD, PhD, ${ }^{d}$ Fausto Biancari, MD, PhD, ${ }^{a}$ and Tatu Juvonen, MD, PhD, ${ }^{a}$ Oulu and Kuopio, Finland

I

n a previous study we have shown the neuroprotective efficacy of fructose-1,6-bisphosphate (FDP) during experimental hypothermic circulatory arrest in pigs. ${ }^{1}$ In that study tissue samples from the left parietal cerebral cortex were obtained for electron microscopy analysis and examined later. Herein, we report the findings of that analysis.

\section{Methods}

Cerebral cortex biopsy specimens of 6 animals from the FDP group and 5 animals from the control group were chosen for electron microscopy analysis. All of these animals survived 7 days after the operation and were electively killed. Details of the experiment are reported in the main article. ${ }^{1}$ The included animals in the FDP group were selected randomly. The cerebral samples (1- to 2-mm-thin sections) from the left parietal cortex were collected after the death of the animal on the 7th postoperative day. The samples were immediately fixed in a mixture of $1 \%$ glutaraldehyde and $4 \%$ formaldehyde in $0.1 \mathrm{~mol} / \mathrm{L}$ phosphate buffer. The sections were further processed, examined, and photographed in a blinded fashion by a senior cell biologist (A.N.), who looked for at least 10 typical representative neurons in each tissue sample. The analysis of the photographic material was performed in a blinded fashion by a senior pathologist (H.T.) using a scoring method presented in the footnote of Table 1. Neuronal mitochondria, nuclei, cytoplasm, and endoplasmic reticulum were analyzed for ischemic injury.

Differences between the study groups have been evaluated by the Mann-Whitney test. Correlation between the histopathologic total score of our previous study and the present ultrastructural neuronal score has been evaluated with the Spearman test.

\section{Results}

The ultrastructural neuronal scores are presented in Table 1. The total neuronal damage score was lower in the FDP group $(P=$

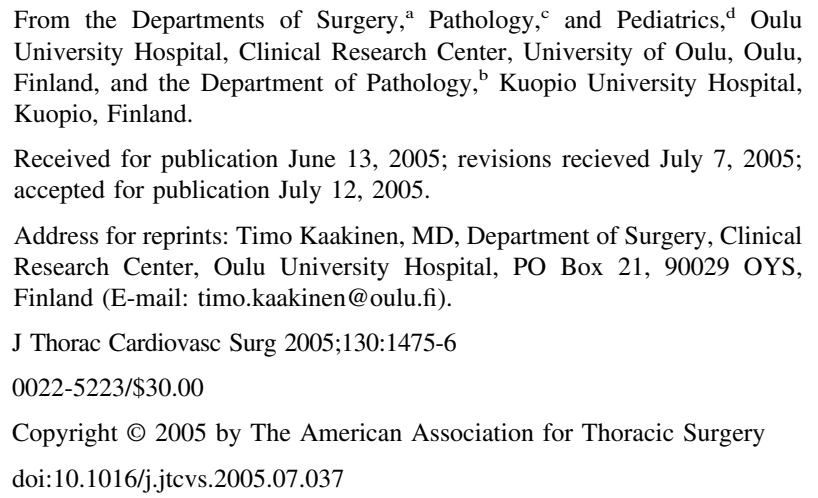

\section{TABLE 1. Neuronal scores}

\begin{tabular}{lccccc}
\hline Protocol & Pig no. & Mitochondria & Nuclei & Cytoplasm & Total score \\
\hline FDP & 1 & 0 & 1 & 1 & 2 \\
& 2 & 2 & 0 & 1 & 3 \\
& 3 & 1 & 1 & 1 & 3 \\
& 4 & 1 & 1 & 1 & 3 \\
& 5 & 2 & 1 & 2 & 5 \\
Mean & 6 & 1 & 0 & 0 & 1 \\
Control & 1 & 1.2 & 0.7 & 1.0 & 2.7 \\
& 2 & 2 & 2 & 2 & 5 \\
& 3 & 2 & 1 & 2 & 5 \\
& 4 & 3 & 2 & 2 & 6 \\
& 5 & 3 & 2 & 2 & 7 \\
Mean & & 2.2 & 1.8 & 2.0 & 7 \\
$P$ value & & .059 & .01 & .011 & .002 \\
\hline
\end{tabular}

The signs of neuronal injury were scored as follows: 0 (normal mitochondria, intact nuclei, no edema in cytoplasm, normal endoplasmic reticulum); 1 (mild-to-moderate mitochondrial edema, chromatin aggregation in nuclei, mild-to-moderate edema in the cytoplasm, swollen but still intact endoplasmic reticulum); 2 (severe mitochondrial edema, disintegrated nuclei, severe cytoplasmic edema, ruptured endoplasmic reticulum); 3 (ruptured mitochondria). From the above scores, a total score was calculated by adding all the scores together. FDP, Fructose-1,6-bisphosphate.

$.002)$, as were the nucleus and cytoplasm scores $(P=.01$ and $P=$ .011 , respectively). The mitochondrion score was somewhat lower in the FDP group $(P=.059)$. There was a significant positive correlation between the histopathologic total score of our previous study and the present electron microscopy total score $(r=0.73$, $P=.01)$.

\section{Discussion}

The key ultrastructural sign of neuronal ischemic injury is mitochondrial swelling with dilation of the intercristal spaces, eventually leading to rupture of the mitochondrial membranes. Membrane rupture releases mitochondrial $\mathrm{Ca}^{2+}$ into the cytoplasm, which, with low adenosine triphosphate concentration, is a major initiating factor of necrotic cell death. The release of mitochondrial proteins, such as cytochrome $\mathrm{c}$ and apoptosis-inducing factor, into the cytoplasm has been shown to induce apoptosis if adenosine triphosphate is still present in the cells in sufficient amounts. Other ultrastructural signs of neuronal injury are clumping of chromatin and nuclear rupture, along with cytoplasmic swelling. Rupture of endoplasmic reticulum and detachment of ribosomes occur after severe injury. These findings indicate loss of capability to synthesize RNA and proteins (Figure 1). ${ }^{2}$ FDP has been found to preserve and resupply cellular energy pools during ischemia- 


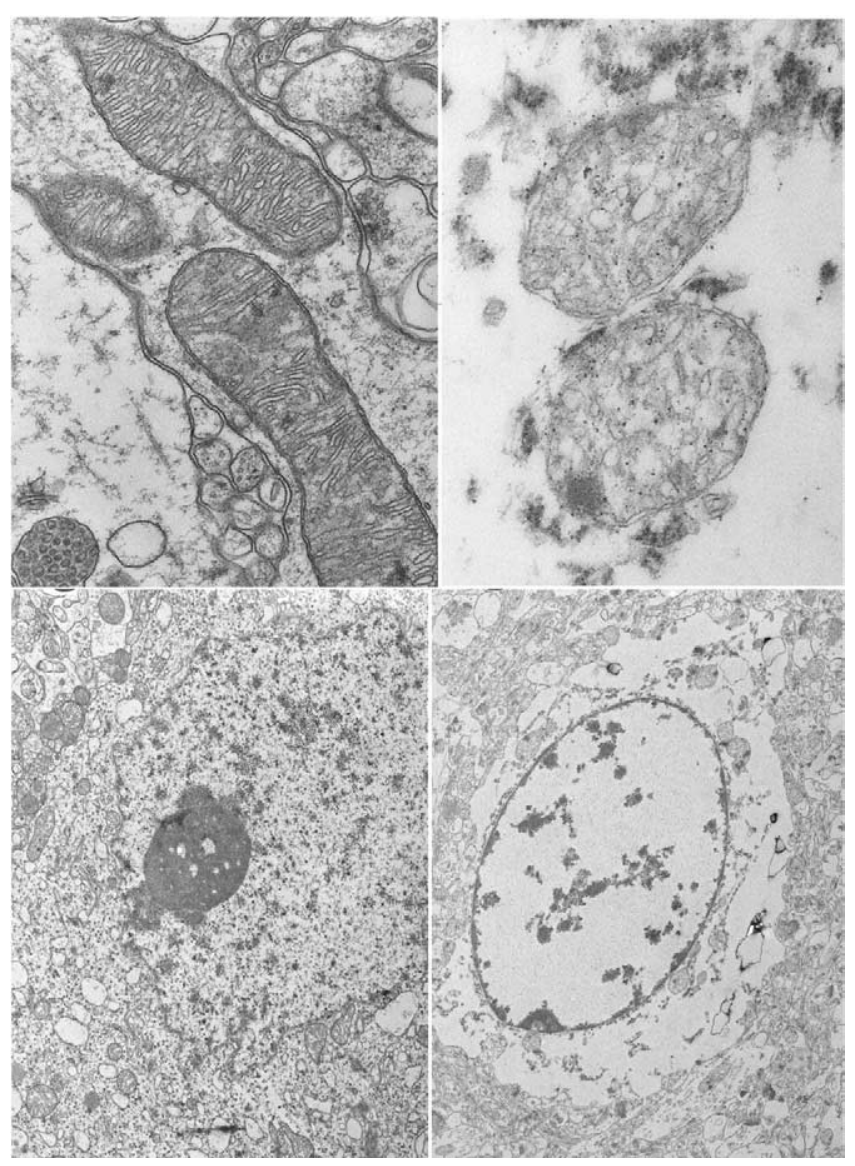

Figure 1. Upper left. Mitochondria with relatively normal intercristal spaces. A sample from the FDP group is shown. (Original magnification $30,000 \times$.) Upper right. Two mitochondria with severe edema, the lower on the verge of membrane rupture. A sample from the control group is shown. (Original magnification $30,000 \times$.) Lower left. A normal nucleus with an intact nucleolus and dispersed chromatin. The cytoplasm is without edema, and endoplasmic reticulum is intact, with ribosomes attached to it. Normal-mildly swollen mitochondria can be seen throughout the cytoplasm. A sample from the FDP group is shown. (Original magnification $5000 \times$.) Lower right: A severely damaged nucleus, with chromatin clumped to the proximity of the membrane. Nucleolus is absent. The cytoplasm is characterized with severe edema. The endoplasmic reticulum is partly ruptured, and detached ribosomes can be seen floating free in the cytoplasm. A sample from the control group is shown. (Original magnification $5000 \times)$. reperfusion injury. ${ }^{3}$ Intracellular increase of $\mathrm{Ca}^{2+}$ during ischemia is inhibited by FDP. ${ }^{4}$ The drug also diminishes the formation of reactive oxygen species during reperfusion. ${ }^{5}$ It seems that FDP might be able to counteract several steps in the mechanism of ischemia-reperfusion injury, a fact that makes it a promising neuroprotective agent. The present ultrastructural findings give further support to the findings of the previous studies indicating neuroprotection by FDP.

The number of animals in the present study is rather low because only 5 animals in the control group survived 7 days. We included in the present study only those animals that survived 7 days after the operation to compare animals with similar clinical outcome and, likely, brains at the same stage after ischemic injury. Despite the small size of the study, a clear difference has been observed between the study groups in terms of neuronal scores, indicating significant neuroprotection by FDP. This is further supported by the fact that the present ultrastructural findings correlated well with the conventional histopathology of our main study on FDP. ${ }^{1}$

In conclusion, the intravenous administration of two $500 \mathrm{mg} / \mathrm{kg}$ doses of FDP before and after hypothermic circulatory arrest in a surviving porcine model is associated with better ultrastructural findings in cortical neurons. These findings further suggest the neuroprotective efficacy of the drug.

We thank Dr Bruno Viglianti, Medical Assistant and Pharmacovigilance Manager, Biomedica Foscama, Ferentino, Italy, for providing us with fructose-1,6-bisphosphate (Esafosfina) and Pasi Ohtonen, MSc, for statistical assistance.

\section{References}

1. Romsi P, Kaakinen T, Kiviluoma K, Vainionpää V, Hirvonen J, Pokela M, et al. Fructose-1,6-bisphosphate for improved outcome after hypothermic circulatory arrest in pigs. J Thorac Cardiovasc Surg. 2003;125: 686-98.

2. Kumar V, Abbas AK, Nelson F. Cellular adaptations, cell injury, and cell death. In: Kumar V, Abbas AK, Nelson F, editors. Robbins and Cotran pathologic basis of disease. Philadelphia: Saunders; 2004. p. 4-46.

3. Tavazzi B, Starnes JW, Lazzarino G, Di Pierro D, Nuutinen EM, Giardina B. Exogenous fructose-1,6-bisphosphate is a metabolizable substrate for the isolated normoxic rat heart. Basic Res Cardiol. 1992;87:280-9.

4. Hassinen IE, Nuutinen EM, Ito K, Nioka S, Lazzarino G, Giardina B, et al. Mechanism of the effect of exogenous fructose 1,6-bisphosphate on myocardial energy metabolism. Circulation. 1991;83:584-93.

5. Vexler ZS, Wong A, Francisco C, Manabat C, Christen S, Tauber M, et al. Fructose-1,6-bisphosphate preserves intracellular glutathione and protects cortical neurons against oxidative stress. Brain Res. 2003;960:90-8. 